\title{
DETERMINATION OF HYGROEXPANSION COEFFICIENTS OF WOOD USING ANALYTICAL AND NUMERICAL HOMOGENIZATION
}

\author{
Lucie Kucíková, Michal Šejnoha*, Jan Vorel \\ Czech Technical University in Prague, Faculty of Civil Engineering, Thákurova 7, 16629 Prague 6, Czech \\ Republic \\ * corresponding author: sejnom@fsv.cvut.cz
}

\begin{abstract}
This paper is concerned with the determination of hygroexpansion coefficients of wood. Wood is a naturally hygroscopic material, attracting moisture from its surroundings. Its dimensions change depending on the actual moisture content. This dependence is characterized by the coefficient of hygroexpansion. For complex microstructures, this coefficient can be determined, similarly to the coefficient of thermal expansion, either from analytical or numerical homogenization. The homogenization procedure, used in this paper, comprises several steps corresponding to the wood structural composition. Accuracy of this approach is governed by correctness of the chosen input parameters. One of the most important parameters are the volume fractions of the earlywood and latewood. Ascertained dependence of the earlywood and latewood volume fractions on dry wood density is also presented.
\end{abstract}

KEYWORDS: Wood, homogenization, hygroexpansion coefficient.

\section{INTRODUCTION}

Environmentally friendly materials are getting to the forefront of global interest and one of them is also wood. The wood is a natural cellular material and as such its properties change depending on growth site and species. Spruce wood, examined in this paper, belongs to conifers and therefore consists mainly of tracheids [1], long closed tube-like cells with cavity inside (called lumen). There are two types of tracheids. The first one is characterized by almost square crosssection and thin cell wall, and it forms the earlywood part of the growth ring. The second one forms the latewood part of the growth ring. Compared to the previous one, its cross-section is almost half in width in the radial direction and the cell wall is much thicker. Generally, a multilayered cell wall structure is simplified to three layers [2]: M layer (middle lamella, primary wall and outer layer of secondary wall $\left.\mathrm{S}_{1}\right), \mathrm{S}_{2}$ layer (middle layer of secondary wall), and $\mathrm{S}_{3}$ layer (inner layer of secondary wall). Each layer is composed of four basic elements: lignin, hemicellulose, cellulose (crystalline and amorphous) and extractives. Due to their low volume fraction, the extractives are often neglected, as was also the case in the present study. The cellulose and hemicellulose are in the form of microfibrils, bonded together by lignin [3]. The microfibrils wound helically along the cell and their deviation from a longitudinal axis is called a microfibril angle (MFA). The microfibril angle generally changes within the cell wall, but for simplicity it is assumed constant in individual layers. Clearly, it is one of the main factors influencing the wood properties, see e.g. 4]

Wood is a naturally hygroscopic material, i.e. it attracts moisture from surrounding atmosphere. The water is present in wood in two forms: free water (i.e. liquid water or water vapor in pores) and bound water (i.e. liquid water in the cell wall). The amount of water present in wood is characterized by the moisture content (MC), defined as the ratio of the mass of water to the mass of oven-dried material. Moisture content at which the cell walls are fully saturated and with no free water in the lumens is called a fiber saturation point (FSP). The average value of FSP is about $30 \%$ [5]. Interestingly, several definitions of fiber saturation point can be found in literature 6]. Below this point, the major part of dimensional changes occurs in the form of shrinkage (contraction) or swelling (expansion).

The resulting moisture strain is linked to the change in moisture content through the hygroexpansion coefficient $\alpha_{h}$ [-]. Estimating this coefficient at the level of wood is the principal objective of this paper. To that end, several homogenization techniques combining classical micromechanics and unit cell simulations will be adopted. These are briefly outlined in Section 3 preceded in Section 2 by microstructure quantification, both in terms of material composition and geometry. The resulting predictions are presented in Section 4 followed by the summary of essential observations in Section 5 .

\section{MATERIALS}

Special attention should be paid to the selection of input parameters used in homogenization. Cell wall constituents are very small particles, which are difficult to measure. In this paper, properties presented 


\begin{tabular}{lccccccc}
\hline & $\begin{array}{c}E_{11} \\
{[\mathrm{GPa}]}\end{array}$ & $\begin{array}{c}E_{22}=E_{33} \\
{[\mathrm{GPa}]}\end{array}$ & $\begin{array}{c}G_{12}=G_{13} \\
{[\mathrm{GPa}]}\end{array}$ & $\begin{array}{c}\nu_{21}=\nu_{31} \\
{[-]}\end{array}$ & $\begin{array}{c}\nu_{22}=\nu_{33} \\
{[-]}\end{array}$ & $\begin{array}{c}\alpha_{h, 11} \\
{[-]}\end{array}$ & $\begin{array}{c}\alpha_{h, 22}=\alpha_{h, 33} \\
{[-]}\end{array}$ \\
\hline Cellulose & 150.00 & 17.50 & 4.50 & 0.01 & 0.50 & 0.000 & 0.000 \\
Hemicellulose & 16.00 & 3.50 & 1.50 & 0.10 & 0.40 & 0.000 & 1.368 \\
Lignin & 2.75 & 2.75 & 1.03 & 0.33 & 0.33 & 0.351 & 0.351 \\
\hline
\end{tabular}

TABLE 1. Phase properties at cell wall level [2].

in [2] were taken as the most relevant and used for computations. Properties of individual cell wall constituents are summarized in Table $\mathbb{1}$ Besides the properties, it is necessary to know the volume fractions of individual constituents within each cell wall layer. These are stated in Table 2, In each layer the hemicellulose and cellulose are deviated by a different microfibril angle. The values of MFA in $\mathrm{M}$ and $\mathrm{S}_{3}$ layers were taken from [2]. The MFA of $\mathrm{S}_{2}$ layer was computed by comparing the values found from homogenization and nanoindentation measurements, see [7.

\begin{tabular}{lccc}
\hline Layer & $\mathrm{M}$ & $\mathrm{S}_{2}$ & $\mathrm{~S}_{3}$ \\
\hline$f_{\text {cellulose }}$ & 0.20 & 0.49 & 0.49 \\
$f_{\text {hemicellulose }}$ & 0.15 & 0.27 & 0.27 \\
$f_{\text {lignin }}$ & 0.65 & 0.24 & 0.24 \\
MFA [ ${ }^{\circ}$ & 45.00 & 32.53 & 75.00 \\
\hline
\end{tabular}

TABLE 2. Volume fraction of cell wall constituents and MFA of individual cell wall layers 2 .

Nanoindentation is a nano-/micro-scale measurement technique based on contact mechanics, usually used to determine the material hardness and elastic modulus. The method grounds on pushing a small hard tip with known geometry and properties to the tested material while simultaneously recording the applied load and the measured displacement. Herein, only the $S_{2}$ layer, as the principal carrier of the cell wall stiffness, was indented using the Berkovich indenter. A certain hold period at the level of maximum force was considered within each loading cycle to eliminate the viscoelastic response of the material. The indentation moduli were then estimated from the unloading part of the loading diagram, see e.g. 8, 9] for more details.

According to the theory of anisotropic indentation presented in [10], the indentation modulus can also be defined in terms of the homogenized stiffness matrix pertinent to the $S_{2}$ layer while taking into account the microfibril angle. Note that the effective stiffness matrix in the local coordinate system can be acquired for example from Eq. (5) adopting the material data from Table 1 and the volume fractions from Table 2 The stiffness matrix entering the theoretical expression of indentation modulus further calls for transformation

\footnotetext{
${ }^{1}$ Axis-1 is oriented along longitudinal (microfibril) direction, axes-22,33 along transverse direction.
}

into the MFA coordinate system. The actual MFA is determined by matching the measured and theoretically calculated indentation moduli as presented, e.g. in 11, 12]. The resulting MFA for $\mathrm{S}_{2}$ layer is listed in Table 2, The distribution of indentation modulus as a function of MFA and the probability density function of MFA derived from a large set of indents are plotted in Fig. 1 for illustration.

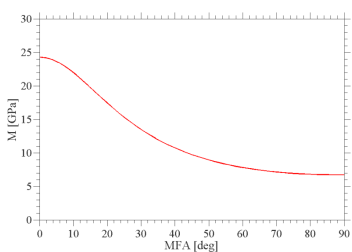

(a)

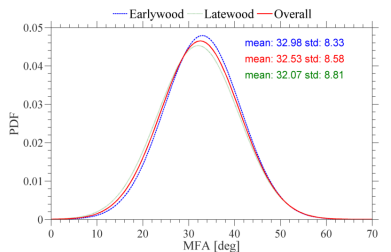

(b)
Figure 1. a) Variation of indentation modulus as a function of MFA, b) Probability density function of MFA

However, a word of caution is in place when adopting the results from nanoindentation measurements directly without any adjustment for potential experimental error. As demonstrated in [13] such a step may significantly underestimate the resulting effective stiffness when compared, e.g. to macroscopic measurements of the effective longitudinal elastic modulus. Consequently, the predicted coefficients of hygroexpansion may also be highly inaccurate. To address this issue we provide, apart from the measured $\mathrm{S}_{2}$ layer $\mathrm{MFA}=32.53^{\circ}$, the results for $\mathrm{MFA}=10^{\circ}, 15^{\circ}, 20^{\circ}$ for the sake of illustration.

The numerical part of homogenization requires the formulation of a periodic unit cell (PUC) representing the material microstructure. Altogether, three types of PUCs were developed to represent the earlywood, latewood, and equivalent wood, while accounting for real microstructural dimensions. The equivalent wood is a combination of both the earlywood and latewood and was created such as to reflect the volume fraction of lumens of the two types of wood within a single growth ring. Apart from that, the volume fractions of individual layers building the cell wall are needed. Herein, we adopt the particular values from [2].

To get the volume fraction of lumens corresponding to an equivalent wood requires the knowledge of the volume fractions of lumens of the earlywood and latewood as well as the volume fraction of earlywood and latewood at the level of growth rings. A potential 
method of attack to derive these quantities is provided by image analysis. Color and binary images of the earlywood of spruce are presented in Figs. 2(a,b) for illustration.
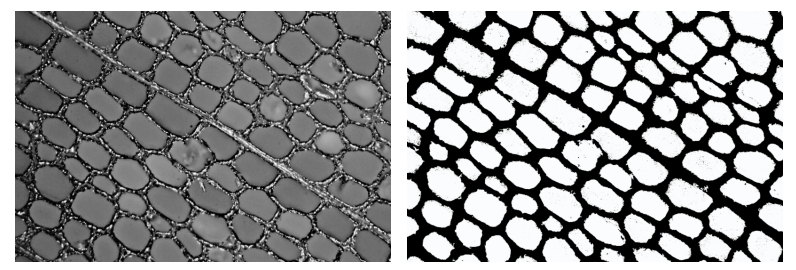

(a)

(b)

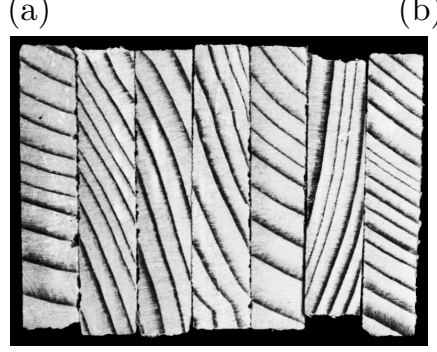

(c)

FiguRE 2. a) Original gray-scale image of earlywood, b) Transformed binary image of earlywood, c) Images of growth rings.

These images were taken at several locations within several growth rings to increase reliability of the estimated volume fractions, at least on the average sense. The variability of microstructure over the length of the growth ring as well as the transition zone (a gradual change between earlywood and latewood) were, therefore, neglected. Fig. 2(c) then offers similar images at the level of growth rings. Considering a constant width of the latewood part equal to $0.2 \mathrm{~mm}$, see also [2], these images allow for obtaining the dependence of the earlywood volume fraction on dry wood density plotted in Fig. 3

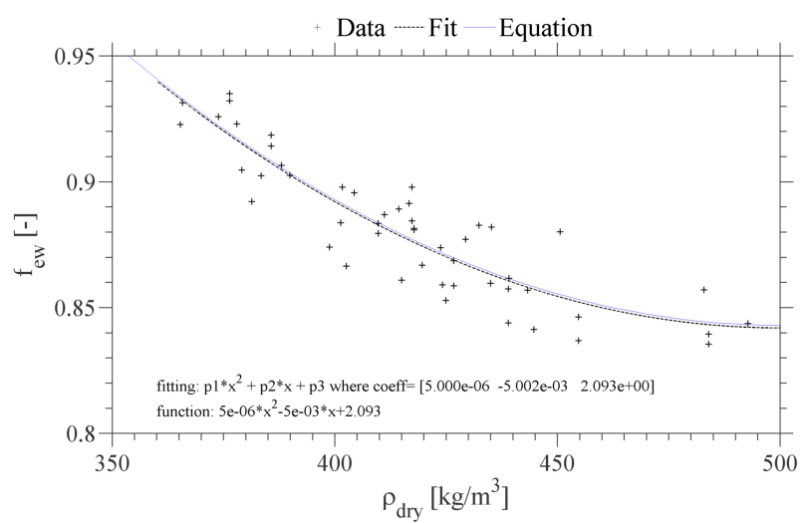

Figure 3. Dependence of the earlywood volume fraction on dry wood density $\left[\mathrm{kg} / \mathrm{m}^{3}\right]$.

Thus, given the dry wood density $\rho_{d r y}\left[\mathrm{~kg} / \mathrm{m}^{3}\right]$ we can estimate the volume fraction of earlywood $f_{E W}$ directly from the fitting equation ${ }^{2}$

$$
f_{E W}=5 \cdot 10^{-6} \cdot \rho_{d r y}^{2}-5 \cdot 10^{-3} \cdot \rho_{d r y}+2.093,
$$

without passing through a tedious measurement process. The volume fraction of latewood is then simply given by

$$
f_{L W}=1-f_{E W} .
$$

It is now possible to define the equivalent wood as basic weighted average of both the earlywood (EW) and latewood (LW). The volume fraction of lumens of equivalent wood $f_{\text {lum }}$ is therefore

$$
f_{\text {lum }}=f_{E W} \cdot f_{\text {lum }, E W}+f_{L W} \cdot f_{\text {lum }, L W},
$$

where $f_{\text {lum, } E W}, f_{\text {lum, } L W}$ are the volume fractions of lumens of earlywood and latewood, respectively. It is now easy to get the desired dimensions of the wood cells.
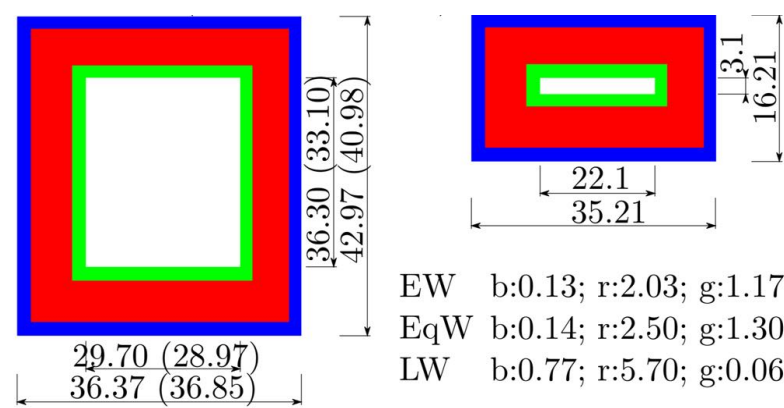

EW b:0.13; r:2.03; g:1.17

EqW b:0.14; r:2.50; g:1.30

LW b:0.77; r:5.70; g:0.06

Figure 4. Periodic unit cells of earlywood, latewood, and equivalent wood.

The resulting periodic unit cells are shown in Fig. 4 When constructing these unit cells we adopted the inner dimensions of lumens, i.e. the dimensions of the inner rectangle of the earlywood and latewood PUCs, from 11. Considering the volume fractions of lumens as $f_{\text {lum }, E W}=0.69$ for the earlywood, $f_{\text {lum }, L W}=0.12$ for the latewood [7], and dry wood density $\rho_{d r y}=390 \mathrm{~kg} / \mathrm{m}^{3}$ allowed us to calculate the cell wall thickness. The volume fractions of individual layers in the cell wall rendered its final subdivision distinguishing again $\mathrm{M}$ layer (blue, b), $\mathrm{S}_{2}$ layer (red, $\mathrm{r}$ ), and $\mathrm{S}_{3}$ layer (green, g). The actual thicknesses are provided in the left bottom corner of the picture for the sake of completeness. The dimensions of the equivalent wood $(\mathrm{EqW})$ were derived in analogy with Eq. (3) as the weighted average of the dimensions of earlywood and latewood, respectively.

The actual arrangement of the unit cells in Fig. 4 may range from perfectly regular to hexagonal array. With reference to Fig. 2 the latter one is considered in the present study. The discretized transverse crosssection of the 3D unit cells are plotted in Fig. 5 Some improvements in the prediction of effective properties

\footnotetext{
${ }^{2}$ Point out that this simple quadratic equation is only applicable for the plotted range of dry wood density $\left(\rho_{d r y} \in(350-500)\right.$ $\left.\left[\mathrm{kg} / \mathrm{m}^{3}\right]\right)$.
} 
can be expected if addressing the microstructure variability more closely [2]. In the light of the present approach this would call for so called statistically equivalent periodic unit cell, which is more refined and matches the actual microstructure in view of twopoint correlation function [14].

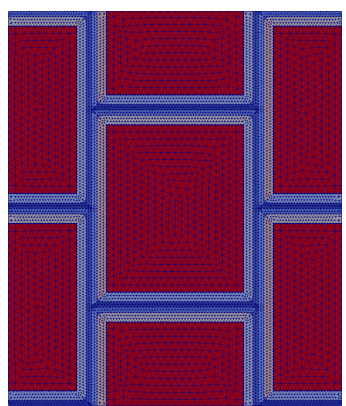

(a)

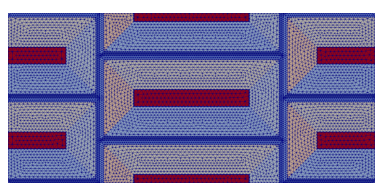

(b)
Figure 5. Geometry and plane view of discretized PUCs: a) earlywood, b) latewood.

\section{Methods}

Wood is often treated as a natural composite. Its structural composition leads to application of hierarchical homogenization, starting from the level of cell wall constituents up to the level of the solid wood. In this case, a combination of analytical and numerical homogenization is used to determine the effective hygroexpansion coefficients. However, according to the formula for the overall strain

$$
\varepsilon=\varepsilon_{e}+\varepsilon_{h}, \quad \varepsilon_{h}=\alpha_{h} u,
$$

where $\varepsilon_{e}$ denotes the elastic strain, $\varepsilon_{h}$ the hygroexpansion strain, $\boldsymbol{\alpha}_{h}$ the vector of hygroexpansion coefficients $3^{3}$ and $u$ the change of moisture content, the effective hygroexpansion coefficients have to be computed simultaneously with the effective mechanical properties.

The computation comprises several steps. The first step corresponds to the level of cell wall layers. Each layer $\left(\mathrm{M}, \mathrm{S}_{2}\right.$, and $\left.\mathrm{S}_{3}\right)$ is composed of three phases, where lignin is considered as a matrix phase and hemicellulose and cellulose as circular cylindrical inclusions. Properties of individual phases are stated in Table 1 and their volume fractions within each layer appear in Table 2. Using the Mori-Tanaka method, one of the most popular analytical methods, we arrive at the estimates of the effective stiffness matrix $\mathbf{L}^{M T}$ in the form

$$
\begin{aligned}
\mathbf{L}^{M T}=\mathbf{L}_{0}+ & \left(\sum_{r=1}^{N-1} f_{r}\left(\mathbf{L}_{r}-\mathbf{L}_{0}\right) \mathbf{A}_{r}^{\text {dill }}\right) \\
& \left(f_{0} \mathbf{I}+\sum_{r=1}^{N-1} f_{r} \mathbf{A}_{r}^{\text {dill }}\right)^{-1},
\end{aligned}
$$

\footnotetext{
${ }^{3}$ Note that $\boldsymbol{\alpha}_{h}$ is assumed constant independent of moisture. Experimental measurements are currently under way to support this assumption.
}

where $\mathbf{L}_{0}$ and $\mathbf{L}_{r}$ are the stiffness matrices of the matrix phase (lignin) and inclusions (hemicellulose, cellulose), $\mathbf{I}$ is the second order identity tensor, $f_{0}$ and $f_{r}$ are the volume fractions of corresponding phases, and $\mathbf{A}_{r}^{\text {dill }}$ is the partial strain concentration factor defined as

$$
\mathbf{A}_{r}^{\text {dill }}=\left(\mathbf{I}+\mathbf{S}_{r} \mathbf{L}_{0}^{-1}\left(\mathbf{L}_{r}-\mathbf{L}_{0}\right)\right)^{-1},
$$

where $\mathbf{S}_{r}$ is the Eshelby tensor depending on the shape of the inclusion.

The effective hygroexpansion coefficients are computed using the Levin formula as

$$
\boldsymbol{\alpha}^{h o m}=\sum_{r=0}^{N-1} f_{r}\left(\mathbf{B}_{r}^{M T}\right)^{T} \boldsymbol{\alpha}_{r},
$$

where $\boldsymbol{\alpha}_{r}$ denotes the vector of hygroexpansion coefficients of individual phases, $\mathbf{B}_{r}^{M T}$ is the stress concentration factor derived again from the Mori-Tanaka method. For individual phases we get

$$
\begin{aligned}
& \mathbf{B}_{0}^{M T}=\left[f_{0} \mathbf{I}+\sum_{r=1}^{N-1} f_{r} \mathbf{B}_{r}^{\text {dill }}\right]^{-1}, \\
& \mathbf{B}_{r}^{M T}=\mathbf{B}_{r}^{\text {dill }} \mathbf{B}_{0}^{M T}, \quad r=1, \ldots, N-1, \\
& \mathbf{B}_{r}^{\text {dill }}=\left[\mathbf{I}+\mathbf{L}_{0}\left(\mathbf{I}-\mathbf{S}_{r}\right)\left(\mathbf{L}_{r}^{-1}-\mathbf{L}_{0}^{-1}\right)\right]^{-1} .
\end{aligned}
$$

Although analytical homogenization may still be exploited even above the cell wall level, we abandon this approach and turn our attention to the periodic unit cell representation of cellular microstructure, recall Fig. 5 This approach builds upon the application of the Hill lemma

$$
\left\langle\delta \varepsilon(\boldsymbol{x})^{T} \boldsymbol{\sigma}(\boldsymbol{x})\right\rangle=\delta \boldsymbol{E}^{T} \boldsymbol{\Sigma},
$$

within the framework of the 1st order homogenization theory. The angle brackets in Eq. (9) denote the volume averaging. Bearing in mind that the evaluation of effective hygroexpansion coefficients is identical to that of thermal expansion, we follow 14 and consider the stress based formulation. We begin with the local constitutive law written as

$$
\boldsymbol{\sigma}(\boldsymbol{x})=\mathbf{L}(\boldsymbol{x})\left(\varepsilon(\boldsymbol{x})-\boldsymbol{\alpha}_{h}(\boldsymbol{x}) u\right),
$$

where $\mathbf{L}$ is the local stiffness matrix, $\boldsymbol{\alpha}_{h}$ is the vector comprising hygroexpansion coefficients and $u$ denotes the moisture content change. Within the 1st order homogenization the local strain $\boldsymbol{\varepsilon}(\boldsymbol{x})$ is split into two parts

$$
\varepsilon(\boldsymbol{x})=\boldsymbol{E}+\varepsilon^{*}(\boldsymbol{x}),
$$

where $\boldsymbol{E}$ is a macroscopic part constant over the PUC and $\varepsilon^{*}(\boldsymbol{x})$ is a fluctuation part, which has to disappear upon volume averaging. This can be achieved, e.g. through the application of periodic boundary conditions [14 in the solution of the discretized system of governing equations 13 . The FEM discretization provides the local strain in the form

$$
\varepsilon(\boldsymbol{x})=\boldsymbol{E}+\mathbf{B}(\boldsymbol{x}) \boldsymbol{r},
$$




\begin{tabular}{lcccccccccccc}
\hline & $\begin{array}{c}E_{11} \\
{[\mathrm{GPa}]}\end{array}$ & $\begin{array}{c}E_{22} \\
{[\mathrm{GPa}]}\end{array}$ & $\begin{array}{c}E_{33} \\
{[\mathrm{GPa}]}\end{array}$ & $\begin{array}{c}G_{23} \\
{[\mathrm{GPa}]}\end{array}$ & $\begin{array}{c}G_{13} \\
{[\mathrm{GPa}]}\end{array}$ & $\begin{array}{c}G_{12} \\
{[\mathrm{GPa}]}\end{array}$ & $\begin{array}{c}\nu_{21} \\
{[-]}\end{array}$ & $\begin{array}{c}\nu_{31} \\
{[-]}\end{array}$ & $\begin{array}{c}\nu_{32} \\
{[-]}\end{array}$ & $\begin{array}{c}\alpha_{11} \\
{[1 / \mathrm{K}]}\end{array}$ & $\begin{array}{c}\alpha_{22} \\
{[1 / \mathrm{K}]}\end{array}$ & $\begin{array}{c}\alpha_{33} \\
{[1 / \mathrm{K}]}\end{array}$ \\
\hline M layer & 34.24 & 4.62 & 3.96 & 1.32 & 1.32 & 1.60 & 0.03 & 0.04 & 0.41 & 0.0246 & 0.3920 & 0.5410 \\
$\mathrm{~S}_{2,3}$ layer & 78.72 & 7.15 & 6.01 & 2.05 & 2.05 & 2.46 & 0.02 & 0.02 & 0.41 & 0.0078 & 0.3247 & 0.5089 \\
\hline
\end{tabular}

TABLE 3. Effective properties at cell wall level from analytical homogenization.

where $\boldsymbol{r}$ represents the nodal displacements of the fluctuation displacement field $\boldsymbol{u}^{*}$. Substituting Eq. (12), combined with Eq. 10, back into Eq. (9) and collecting terms associated with $\delta \boldsymbol{r}$ and $\delta \boldsymbol{E}$ finally gives the aforementioned discretized system of algebraic equations in the form

$$
\begin{aligned}
& {\left[\begin{array}{cc}
\int_{\Omega} \mathbf{L} \mathrm{d} \Omega & \int_{\Omega} \mathbf{L} \mathbf{B} \mathrm{d} \Omega \\
\int_{\Omega} \mathbf{B}^{T} \mathbf{L} \mathrm{d} \Omega & \int_{\Omega} \mathbf{B}^{T} \mathbf{L} \mathbf{B} \mathrm{d} \Omega
\end{array}\right]\left\{\begin{array}{c}
\boldsymbol{E} \\
\boldsymbol{r}
\end{array}\right\}=} \\
& \left\{\begin{array}{c}
\Omega \boldsymbol{\Sigma}+\int_{\Omega} \mathbf{L} \boldsymbol{\alpha}_{h} u \mathrm{~d} \Omega \\
\int_{\Omega} \mathbf{B}^{T} \mathbf{L} \boldsymbol{\alpha}_{h} u \mathrm{~d} \Omega
\end{array}\right\},
\end{aligned}
$$

with the macroscopic strain $\boldsymbol{E}$ and the nodal fluctuation displacements $\boldsymbol{r}$ as unknown quantities. Solving the system (13), while setting the macroscopic stress $\boldsymbol{\Sigma}=\mathbf{0}$ and the moisture change $u=1$, provides the effective hygroexpansion coefficients as components of the macroscopic strain $\boldsymbol{E}$. This step takes into account the microfibril angle as a deviation of inclusions from the longitudinal axis. Thus for each segment along the perimeter of the cell wall a proper transformation of coordinates from local to global coordinate system is needed 2 .

If using the PUC corresponding to equivalent wood we get the effective coefficients of hygroexpansion directly at the level of the solid wood. On the contrary, employing the unit cells pertinent to the earlywood and latewood requires an additional homogenization step, where the corresponding effective stiffness matrices are also needed. This can be achieved by setting $u=0$ in Eq. 13 and load the unit cell in turn by each of the six components of $\boldsymbol{\Sigma}$ set equal to one to get individual columns of the $6 \times 6$ compliance matrix $\mathbf{M}^{\text {hom }}$ as corresponding strain volume averages. The required effective stiffness matrix $\mathbf{L}^{\text {hom }}$ then follows from simple matrix inversion.

Once having the effective stiffnesses of the earlywood and latewood we may proceed with the derivation of $\alpha^{\text {hom }}$ at the level of solid wood. In such a case one may accept the similarity between the structure of growth rings and a laminate and adopt classical lamination theory, see e.g. [15]. Nevertheless, we proceeded in the footsteps of the previous calculations and performed FEM homogenization of a two layer PUC where individual layers represent the earlywood and latewood, respectively.

\section{Results}

Evaluation of effective coefficients of hygroexpansion of wood required several computational steps on individual scales. The homogenized elastic stiffnesses and coefficients of hygroexpansion of individual layers of the cell wall were determined first using the Mori-Tanaka method, particularly Eqs. (5) and (7). The resulting effective properties assuming $\mathrm{MFA}=0$ in all layers are stored in Table $3 \|^{2}$ As these refer to the level of cell wall, they are the same for earlywood, latewood and equivalent wood.

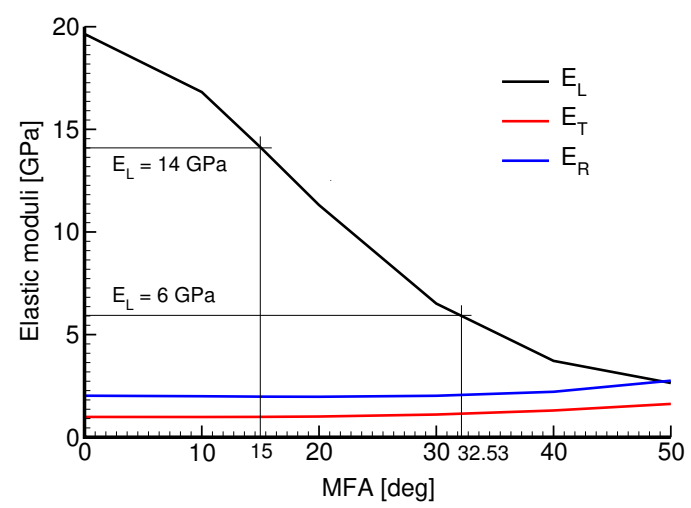

(a)

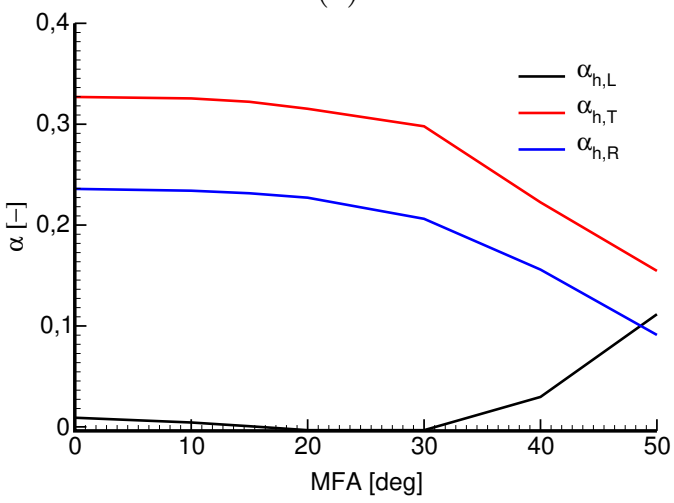

(b)

FiguRE 6. Variation of selected homogenized properties as a function of MFA: a) elastic moduli, b) coefficients of hygroexpansion.

The effective coefficients of hygroexpansion of earlywood, latewood and equivalent wood followed directly from the application of Eq. 13 at the level of lumens. Therein, the properties from Table 3 were properly adjusted to account for the variation of MFA in individual cell wall layers, recall Table 2. When adopting the earlywood and latewood PUCs in Fig. 5 we exercised Eq. 13) one more time at the level of growth rings on a laminate-like PUC. For illustration we plot the variation of the selected homogenized moduli and

\footnotetext{
${ }^{4}$ Axis-1 is oriented in longitudinal direction, axis- 2 in circumferential direction, axis- 3 in cell wall thickness direction.
} 
coefficients of hygroexpansion as a function of $\mathrm{MFA}^{5}$. More specifically, the MFA in $\mathrm{S}_{2}$ layer was varied from $0^{\circ}$ to $50^{\circ}$ while keeping the MFA values from Table 2 for the $\mathrm{M}$ and $\mathrm{S}_{3}$ layers.

Based on the results from simple tensile tests the expected MFA would range from $10^{\circ}$ to $20^{\circ}$. As seen in Fig. 6(a) the value of $\mathrm{MFA}=32.53^{\circ}$ would greatly underestimate the wood stiffness. Thus to compare the resulting predictions of the homogenized coefficients of hygroexpansion provided by the 3 step (cell wall level, level of lumens, level of annual rings) and 2 step (cell wall level, level of lumens assuming equivalent wood) homogenization we set $\mathrm{MFA}=15^{\circ}$. The corresponding results are available in Table 4 . Comparing the last two rows it can be seen that the equivalent wood PUC can safely be used in homogenization analyses thus skipping the last homogenization step at the level of annual rings.

\begin{tabular}{lccc}
\hline & $\alpha_{h, L}$ & $\alpha_{h, T}$ & $\alpha_{h, R}$ \\
\hline Earlywood & 0.002 & 0.34 & 0.21 \\
Latewood & -0.003 & 0.32 & 0.39 \\
Solid Wood & 0.001 & 0.33 & 0.23 \\
Equivalent wood & 0.001 & 0.34 & 0.23 \\
\hline
\end{tabular}

TABLE 4. Effective coefficients of hygroexpansion of wood from numerical homogenization $\left(\mathrm{MFA}=15^{\circ}\right)$.

\section{Conclusions}

In this paper the Mori-Tanaka micromechanical scheme was effectively combined with numerical simulations based on the 1st order homogenization theory. While the Mori-Tanaka method provided the effective properties of individual cell wall layers, numerical homogenization was adopted at the level of tracheids and annual rings. In addition, an equivalent wood was introduced in order to avoid the last homogenization step at the level of annual rings, which is needed when the earlywood and latewood are considered independently. This will prove useful when modeling the evaluation of moisture content in wood through the application of multi-Fickian moisture transport model [16, 17]. As seen in Table 4, the results for the solid wood and equivalent wood are very similar. This clearly promotes the applicability of equivalent wood and thus the derivation of effective properties through a single homogenization step above the cell wall level. Inadequacy of using MFA obtained directly from nanoindentation was also mentioned.

\section{ACKNOWLEDGEMENTS}

The financial support provided by the GAČR grant No. 1805791S and by the Czech Technical University in Prague within SGS project with the application registered under the No. SGS17/168/OHK1/3T/11 is gratefully acknowledged.

\footnotetext{
${ }^{5} \mathrm{~L}$ stands for longitudinal (lumen) direction, $\mathrm{T}$ for tangential direction, $\mathrm{R}$ for radial direction.
}

\section{REFERENCES}

[1] P. O. Kettunen. Wood: Structure and Properties. Trans Tech Publications Ltd, Uetikon-Zuerich, 2006.

[2] K. Persson. Micromechanical Modelling of Wood and Fibre Properties. Ph.D. thesis, Lund University, 2000.

[3] H. E. Desch, J. M. Dinwoodie. Timber: structure, properties, conversion and use. 7th. Macmillan Press, Hampshire, 1996.

[4] L. Donaldson. Microfibril angle: measurement, variation and relationships-a review. Iawa Journal 29(4):345-386, 2008.

[5] R. J. Ross, et al. Wood handbook: Wood as an engineering material. USDA Forest Service, Forest Products Laboratory, General Technical Report FPL-GTR-190, 2010: 509 p 1 v 190, 2010.

[6] E. T. Engelund, L. G. Thygesen, S. Svensson, C. A. Hill. A critical discussion of the physics of wood-water interactions. Wood science and technology 47(1):141-161, 2013.

[7] L. Kucíková. Micromechanical study of spruce wood. Master's thesis, Czech Technical University in Prague, 2018.

[8] W. C. Oliver, G. M. Pharr. Measurement of hardness and elastic modulus by instrumented indentation: Advances in understanding and refinements to methodology. Journal of materials research 19(1):3-20, 2004.

[9] M. L. Oyen, R. F. Cook. A practical guide for analysis of nanoindentation data. Journal of the mechanical behavior of biomedical materials 2(4):396-407, 2009.

[10] J. Vlassak, M. Ciavarella, J. Barber, X. Wang. The indentation modulus of elastically anisotropic materials for indenters of arbitrary shape. Journal of the Mechanics and Physics of Solids 51(9):1707-1721, 2003.

[11] A. Jäger, T. Bader, K. Hofstetter, J. Eberhardsteiner. The relation between indentation modulus, microfibril angle, and elastic properties of wood cell walls. Composites Part A: Applied Science and Manufacturing 42(6):677-685, 2011.

[12] M. Šejnoha, L. Kucíková, J. Vorel, et al. Comparing nano and macroindentation in search for microfibril angle in spruce. Internationl Journal of Computational Methods and Experimental Measurements 5(2):135-143, 2017.

[13] T. Janda, L. Kucíková, J. Vorel, et al. Bayesian inference as a tool for improving the prediction of effective elastic properties of wood. Advances in Engineering Software 2018. Submitted.

[14] M. Šejnoha, J. Zeman. Micromechanics in Practice. WIT Press, Southampton, Boston, 2013.

[15] G. W. Milton. The Theory of Composites. Cambridge Monographs on Applied and Computational Mathematics. Cambridge University Press, 2002. DOI:10.1017/CBO9780511613357.

[16] K. Krabbenhoft, L. Damkilde. A model for non-fickian moisture transfer in wood. Materials and Structures 37(9):615-622, 2004.

[17] H. L. Frandsen, L. Damkilde, S. Svensson. A revised multi-Fickian moisture transport model to describe non-Fickian effects in wood. Holzforschung 61(5):563-572, 2007. 